\title{
Evaluation Methods in Systems Research
}

\author{
Olexandr Polishchuk \\ Laboratory of Modeling and Optimization of Complex Systems, \\ Pidstryhach Institute for Applied Problems of Mechanics and Mathematics, \\ National Academy of Sciences of Ukraine \\ Lviv, Ukraine \\ e-mail: od polishchuk@ukr.net
}

\begin{abstract}
Purpose, subject and main problems of complex dynamical systems evaluation are described. Methods of versatile deterministic evaluation based on local, forecasting, aggregated, and interactive analysis of the state, operation quality, and interaction of complex hierarchical network system's elements on the all hierarchical levels are proposed.
\end{abstract}

Index Terms-Complex System, Network, Evaluation, Forecasting, Aggregation, Continuous Monitoring

\section{INTRODUCTION}

Complex system with hierarchically-network structure are used almost in all areas of human activity, e.g. in transportation, supply and logistics, information and communication, in economics, finance, education, healthcare etc. Their state and functioning quality impose large impact on citizens' quality of life, efficiency of economy and possibilities for its development, readiness of government structures to mitigate impacts of technological and natural disasters $[1,2]$. Finally, they may be treated as the evidences of country development level in general. Complex systems appear, operate and develop within long periods of time and with natural processes of "aging", despite regular improvements, more strict and accurate control over their behaviour is required $[3,4]$. This is why the development of methods for evaluation and forecasting the state, operation quality and interaction between structural elements of complex systems, presented in this work, takes especially important place.

Theory of evaluation of complex systems is a component of the system analysis [5]. On the other hand, evaluation results are objective and the most significant reason for making an informed decision regarding further action on the studied system [6, 7]. In this information content of evaluation, its understandability, and convenient procedures for operational orientation in a large number of obtained conclusions allow to make timely organizational and management decisions [8].

\section{COMPlEX NeTwORKS AND NETWORK SySTEMS}

In the recent years, the theory of complex networks is developing rapidly [9]. The term "network" usually stands for the set of nodes connected by some relations or edges (if graphic representation is available). Complex network is often called "the system". However, let us imagine a railway network as a set of stations and railway tracks connecting them where the railway traffic is absent or a gas or oil supply network as a set of compressor stations and pipelines connecting them which are not pumped with gas or oil. How would computer network represented as a set of servers, computers and wire or wireless connection means look like, if it wasn't used for exchange of information? Lot of further examples may be provided. Presence of material and/or information flows is one of the features that characterize certain object as a system $[10,11]$. The purpose of creation, operation and development of any network is to provide the motion of flows, i.e. it is the motion of flows that makes complex network a system. In this case, network is only a "frame" of the system.

All network systems (NWS) regardless of their type and purpose can be differentiated according to flow organization. We distinguish systems with three levels of organization: fully ordered motion of flows, partially ordered motion of flows and disordered motion of flows. Railway transport system of country and power supply systems are examples of NWS with fully ordered motion of flows. Road transport system of large city or country, postal and trade networks are examples of NWS with partially ordered motion of flows. Social networks, electronic media, mobile communication systems, etc. are examples of NWS with disordered motion of flows. Complex networks with the same structure and different levels of ordered motion of flows generate different network systems.

Organization of flows must be maintained. This means that control of state, operation quality and interaction between objects that comprise the system and provide motion of flows in the network is required. Such functions are performed by control system of NWS. Control system can be organized according to territorial, operational or hybrid principle. Control system and NWS controlled by it together form hierarchical network system (HNWS). Most of industrial, transportation, financial and other systems created and controlled by human kind are the HNWSs. Specific feature of HNWS is that each subsystem of some hierarchal level is divided into a set of subsystems that create subnetwork of the network of lower level [10]. At each level of the hierarchy, the edges ensure smooth motion of flows of certain type, whereas the nodes ensure their processing. The lowest level of hierarchy provides motion of flows for which the network was 
created (trains, cars, energy resources, information etc.). Information, organizational and administrative decisions, cash flows, etc. are flows of higher levels of hierarchy (control levels).

Different ordering of motion of flows requires usage of different approaches to the analysis of network system operation process. These approaches can be based on deterministic, statistical, stochastic and hybrid principles [1, 7]. Each of them has its own advantages and disadvantages [12]. Purpose of deterministic methods consists in formulation of conclusions regarding actual state and operational quality of each system element (node, edge, flow). It is often impossible to perform detailed analysis of all objects of the system. For example, comprehensive medical examination of all citizens of the country is very difficult to carry out. It is also impossible to track the traffic of all vehicles in megalopolis. At the same time, such researches are extremely important for planning production and procurement of medications and medical devices or for improvement of efficiency of transport system in large cities. In such cases, the statistical methods are used. The validity of statistical studies of general set depends on thoroughness of the deterministic analysis of objects included in the representative set If information about system is incomplete or unclear, the stochastic methods are applied. In this case, preliminary data on the distribution of probabilities are usually obtained from results of statistical studies. The history of nuclear power started over half the century ago. At the beginning of its development, it was estimated that the probability of a serious nuclear reactor accident comprises 1 accident in 10 million years [12]. During the operation of nuclear reactors there were at least 15 serious accidents and catastrophes. The number of nuclear reactors operating throughout the word reached 438, and their total operational time is by times lower than 10 million years. It should also be taken into account that there are many systems that require usage of solely deterministic research methods. Indeed, using probabilistic approach to diagnostics or airliner onboard systems check doesn't seem appropriate. Deterministic methods of research are used for analysis of all NWSs, regardless of the level of ordered motion of flows, however the scope and purpose of these studies are different.

\section{Purpose, SubJeCt And Main Problems of Complex NETWORK SYSTEMS EVALUATION}

Usually, the most popular purpose of evaluation consists in the search of system objects whose operation is unsatisfactory [13]. These objects have negative impact on all related system components. Operation of HNWS may be significantly improved through improvement of such objects. Search of system objects that operate perfectly is also important. Those objects may be then used as references. Expanding of principles of their operation organization to other similar objects of HNWS also contributes to improvement of its general operation. Novelty detection is the purpose of the study of some systems. This means for example search for atypical objects on the basis of satellite, GPR or aerial images
[14]. The subject of evaluation of each system object of any hierarchy level is its

- state;

- operational quality;

- efficiency of interaction with other objects of system.

These signs are interrelated and mutually dependent. Indeed, it is difficult to expect high-quality operation of object, if its state is unsatisfactory. Objects that operate unsatisfactory have negative impact on all system elements interacting with them. Combination of the results of evaluation of the state and operational quality of object as well as its interaction with other components provides a fairly complete and integral idea of object operational quality.

Let us list main problems that involve evaluation of complex systems:

1. Evaluation of state and operational quality of system element. Solution for this problem allows to determine elements representing potential threat for operation of system in general and those capable of causing failures as well as to analyse their impact on surrounding elements. If system is consists of elements of the same type, solution of this problem allows to determine elements operating in the best way, i.e. reference elements. Finally, development of generalized conclusions regarding general system operation quality is based on results of evaluation of system elements [15].

2. Forecasting state and operational quality of system elements. State and operational quality of element usually changes in course of time. These attributes can cross "safety threshold" and pose danger to separate components of the system [16]. Forecasting the behavior of the evaluations may be short- and long-term. In any case forecasting term must provide possibility to correct possible faults [17].

3. Choice of optimal mode for system operation. Solution of this problem allows to determine both most appropriate and extreme system operating modes, as well as modes of potential failure [18].

4. Evaluation of system state and operational quality. Solution of this problem allows to determine general quality of system operation according to defined set of parameters, criteria and operating modes [19].

5. Choice of optimally operating system from given class of equivalent systems. Solution of this problem allows to determine the best (reference) or the worst systems in class. Optimally operating elements, modes and systems determined during evaluation may be used as practically reachable quality references [20].

6. Analysis of system operation history. Solution of this problem allows to track and forecast the quality of system operation, to determine trends of its development in the context of improvement or deterioration and to prevent possible failures [12]. 
List of evaluation problems for each particular system can be expanded with regard to its features and purpose of the research [21].

\section{Methods For Evaluation of COMPLEX Network SYSTEMS}

Usually, two main approaches are applied to control state and behavior of existing regional HNWS: regular scheduled inspections, distinctive features of which are accuracy and possibility for further development of recommendations for elimination of drawbacks discovered [22]; and continuous monitoring of system objects' functioning that allows us to draw mediate, but still significant conclusions regarding its actual state and functioning quality [23].

It is reasonable to start evaluation of real systems with objects of lowest structural level, i.e. with elements of HNWSs. We define an element as an object of clearly defined location, functional destination and relevant set of characteristics describing its state and functioning process with corresponding ranges of permissible values for those characteristics. All characteristics are evaluated according to certain collection of criteria and parameters. Of course, evaluation of every object presupposes evaluation of its state on the first place, and only after that the evaluation of quality of implementation of its functions that in any case depend on element's state - either directly or indirectly. The process of evaluation is started only after the stage of thorough selection and processing of experimental data as to each of characteristic and their conversation into format, suitable for further analysis.

Currently, for evaluation of HNWS integer rating or conceptual ("excellent", "good", "satisfactory", "unsatisfactory") scale [13] is commonly used. Its main drawback is that "satisfactory" evaluation may imply wide range of concepts - from "almost good" to "slightly better than unsatisfactory". We propose $[15,17]$ unified approach for evaluating state, quality of functioning and interaction between system structural elements, which consists in developing main rating evaluation and its adjustment with regard to type and features of object studied. Such an approach allows not only to compose more clear understanding of evaluated object, but also to localize the reasons for drawbacks discovered.

The number of characteristics describing element may comprise dozens [8]. Different characteristic may be selected in different ways and they priority regarding structure and functions of element may be different. It is clear that the conclusions as to separate characteristics are to be generalized with consideration of their priority. Recording the number of actually evaluated elements' characteristics is also important. From this point on, evaluations for elements' state and functions they implement on the basis of their characteristics behavior analysis will be referred to as local [17].
As usual, scheduled inspections of system's objects are held at different time points, which means the results of last study may not stay on such stage till following inspection, and state of object and its functioning quality may cross "safety threshold". It should be also taken into account that every real system evolves in time, i.e. with regard to current requirements, its evaluation may be insufficient. Therefore, evaluation process should contain means of analysis of systems meeting expected requirements for short- and longterm perspective. Thus, the evaluation process should not only determine conclusions and discover "faulty" elements for the time point moment when study is held, but also it should forecast further behavior of system objects. Forecasting analysis performed on the basis of local evaluations prehistory, allows us to determine the nature, direction and speed of system state change, follow up negative processes and forecast potential risks, as well as material and financial expenses required for their elimination or timely prevention [17].

Number of local evaluations of real HNWS may reach dozens of millions values [19], which obviously exceed the capacity of their manual analysis. For their generalization, i. e. for developing conclusions regarding their state, quality of functioning and interaction of objects of higher hierarchy levels (subsystems and HNWS in general), tools of linear and non-linear aggregation are applied [15], taking into account weighted coefficients that reflect importance of separate objects in system's structure and priority of functions they perform. Since weighted averaging mitigates the results of both positive and negative evaluations, it is reasonable to make generalization of conclusions after elimination of causes and revaluation of drawbacks eliminated. Let us refer to above described method as to aggregated [19].

Due to the number of reasons, scheduled inspections may often not discover drawbacks that arise "out of schedule". It should be also taken into account that even excellent state and functioning quality of separate objects in the system do not ensure high performance of its subsystems or system in general. And vice versa, the most optimal work organization process will not ensure high efficiency of system functioning if HNWS's state or organization of components functioning is unsatisfactory. The more worn-out HNWS's objects are the more urgent is the problem of continuous monitoring of their state and functioning process [23, 24]. Quality of implementation of functions by object may be affected by number of third-party factors, both internal and external as to the system. Internal influence may be evaluated on the level of subsystems connecting interacting objects. We shall call this evaluation method interactive [25]. It allows us to determine separate objects in selected subsystem, functioning of which is unsatisfactory, without thorough analysis of state and functioning quality of these objects and expenses related to such analysis. The simplest interactive evaluation may be performed for system where the movement of flows is deterministic, at least partially, in accordance with certain 
schedule, the compliance to which may be periodically summed up. It is reasonable to include generalized results of interactive evaluation over certain time period between two scheduled inspections into aggregated evaluation procedure. Those results may be also used for more detailed and accurate forecasting analysis of functioning of evaluated system's objects.

In general, only if combined, proposed methods may provide sufficiently full and adequate understanding of HNWS quality. Indeed, high local evaluations do not ensure effective interaction of elements, failures of separate systems objects may result in breakdown in balanced organization, satisfactory state of object for the moment of current inspection does not imply the state will stay satisfactory till the next inspection. Huge amount of information regarding separate HNWS elements without appropriate generalization is ill-suited for rapid analysis and timely reaction for drawbacks discovered. On higher generalization levels, evaluation allows to determine reliable conclusion as to the state and functioning quality of system and its main subsystems and to define measures, as well as material and finance expenses required for its modernization and optimization of functioning. At the local level evaluation allows to identify separate elements and their components subject to improvement.

\section{CONCLUSIONS}

This work proposes methodology for complex evaluation of real large complex dynamical system with hierarchicallynetwork structure, the component of which are the methods for local, forecasting, interactive and aggregated evaluation of its main objects. It is showed that when combined, together with use of adjusted rating scale they form up quite comprehensive, adequate, and integral notion regarding state, operation quality and interaction of objects of studied system and its subsystems on all levels of its structure. Described methodology is applied for development of software for evaluation of state and functioning quality of track and station facilities of Ukrainian Railways. Separate methods of proposed methodology were applied for evaluation of quality of prosthesis of lower limbs of disabled persons and level of recovery of functional capabilities of human musculoskeletal system at different cases of pathologies and means of rehabilitation which indicates universality of proposed approach for complex systems evaluation. Results of the evaluations should be used to make decision about how to proceed with regard to the objects of estimation.

\section{REFERENCES}

[1] G. E. Alcorta and P.M. Frank, "Deterministic nonlinear observer-based approaches to fault diagnosis: A survey", Control Engineering Practice, vol. 5 (5), pp. 663-670, May 1997.

[2] C. L. Hwang, F. A. Tillman, and M. N. Lee, "System-Reliability Evaluation Techniques for Complex/ Large Systems. A Review", IEEE Transactions, Reliability, vol. 30 (5), pp. 416-423, Dec. 1981.

[3] Y. Bar-Yam, "About Engineering Complex Systems: Multiscale Analysis and Evolutionary Engineering", Engineering Self-Organising Systems: Methodologies and Applications, London: Springer, pp. 16-31, Aug. 2005.
[4] D. Roy and T. Dasgupta, "Evaluation of reliability of complex systems by means of a discretizing approach", International Journal of Quality \& Reliability Management, vol. 19 (6), pp. 792-801, Sept. 2002.

[5] C. L. Owen, "Evaluation of complex systems", Designe Studies, vol. 28 (1), pp. 73-101, Jan. 2007.

[6] G. M. Marakas, Decision support systems in the 21st Century, Cambridge: Pearson Education, 2003.

[7] K. Claxton, "The irrelevance of inference: a decision-making approach to the stochastic evaluation of health care technologies", Journal of Health Economics, vol.18 (3), pp. 341-364, June 1999.

[8] J. Lee and C.-C. Jay Kuo, "Tree model simplification with hybrid polygon/billboard approach and human-centered quality evaluation", 2010 IEEE International Conference on Multimedia and Expo, IEEE Xplore, pp. 932-937.

[9] S. Boccaletti et all., "Complex networks: Structure and dynamics", Physics reports, vol. 424 (4), pp. 175-308, Apr. $2006 .$.

[10] D. Polishchuk, O. Polishchuk, and M. Yadzhak, "Complex deterministic evaluation of hierarchically-network systems: I. Methods description", System Research and Information Technologies, vol. 1, pp. 21-31, Mar. 2015.

[11] O. Polishchuk, D. Polishchuk, "Monitoring of flow in transport networks with partially ordered motion", XXIII Conf. Carpenko physics and mechanics institute, NASU, 2013, pp. 326-329.

[12] D. Polishchuk, O. Polishchuk, and M. Yadzhak, "Comparison of methods of complex system evaluation", Information Extraction and Processing, vol. 32 (108), pp. 110-118, July 2010.

[13] M. Bloom, J. Fischer, and J. Orme, Evaluating practice, Boston: Allyn and Bacon, 2006.

[14] M. Q. Patton, How to use qualitative methods in evaluation, New York: Sage Publications, 1987.

[15] D. Polishchuk and O. Polishchuk, "About evaluation of complex dynamical systems", Journal of Complex Systems, Article ID 204304, Available: http://dx.doi.org/10.1155/2013/ 204304, 6p., Sept. 2013.

[16] A. D. Polishchuk,. "About convergence the methods of projections for solution potential theory integral equation", Computer centre of Siberian Division of AS of the USSR: Preprint 776, 1988.

[17] D. Polishchuk, O. Polishchuk, and M. Yadzhak, "Complex deterministic evaluation of hierarchically-network systems: II. Local and forecasting evaluation", System Research and Information Technologies, vol. 2, pp. 26-38, June 2015.

[18] O. Polishchuk, "Choice of optimal regimes for functioning of complex dynamical systems", Mathematical Methods and Physicomechanical Fields, vol. 48 (3), p. 62-67, Sept. 2005.

[19] D. Polishchuk, O. Polishchuk, M. Yadzhak, Complex deterministic evaluation of hierarchically-network systems: III. Aggregative evaluation", System Research and Information Technologies vol. 4, pp. 20-31, Dec. 2015.

[20] O. Polishchuk, "About the choice of the optimal dynamical system from the class of equivalent systems", Information Extraction and Processing, vol. 20 (96), pp. 23-28, Dec. 2004.

[21] F.-Y. Wang, "Computational experiments for behavior analysis and decision evaluation of complex systems", Journal of System Simulation, vol. 16 (5), pp. 893-897, May 2004.

[22] O. Polishchuk, M. Tyutyunnyk, and M. Yadzhak, "Quality evaluation of complex systems function on the base of parallel calculations", Information Extraction and Processing, vol. 26 (108), pp. 121-126, Dec. 2007.

[23] T. Lung-Wen, Distributed real time systems: monitoring, visualization, debugging and analysis, Paris: Lavoisier, 1996.

[24] F. Zhao, X. Koutsoukos, H. Haussecker, J. Reich, and P. Cheung, "Monitoring and fault diagnosis of hybrid systems", IEEE Transactions on Systems, Man, and Cybernetics, vol. 35 (6), pp. 1225-1240, Dec. 2007.

[25] D. Polishchuk, O. Polishchuk, M. Yadzhak, Complex deterministic evaluation of hierarchically-network systems: IV. Interactive evaluation", System Research and Information Technologies vol. 1, pp. 7-16, Mar. 2016. 